Bundesgesundheitsbl 2016 · 59:396-403

DOI 10.1007/s00103-015-2303-6

Online publiziert: 11. Januar 2016

(c) Springer-Verlag Berlin Heidelberg 2015

CrossMark

\author{
Birte Bödeker ${ }^{1,2}$. Linda Seefeld ${ }^{3}$. Stephanie Buck ${ }^{1}$. Oliver Ommen ${ }^{3}$. \\ Ole Wichmann ${ }^{1}$ \\ ${ }^{1}$ Fachgebiet Impfprävention, Abteilung für Infektionsepidemiologie, \\ Robert Koch-Institut, Berlin, Deutschland \\ ${ }^{2}$ Charité - Universitätsmedizin Berlin, Berlin, Deutschland \\ ${ }^{3}$ Referat 1-11, Bundeszentrale für gesundheitliche Aufklärung, Köln, Deutschland
}

\title{
Wie werden die Impfempfehlungen gegen saisonale Influenza und gegen humane Papillomaviren in gynäkologischen Praxen umgesetzt?
}

\section{Hintergrund und Fragestellung}

Schwangere und ihre Neugeborenen haben ein erhöhtes Risiko für influenzabedingte Komplikationen und damit einhergehende Hospitalisierungen [1,2]. Darüber hinaus kann eine Influenzaerkrankung in der Schwangerschaft die Gefahr einer Fehl-, Früh- oder Totgeburt erhöhen und das Auftreten möglicher Wachstumsverzögerungen sowie ein geringeres Geburtsgewicht beim Neugeborenen begünstigen [3]. In vielen Studien wurde die Sicherheit und Wirksamkeit der Impfung sowohl für Schwangere als auch für das Kind belegt [4-6]. Daher empfiehlt die Ständige Impfkommission (STIKO) seit 2010 die saisonale Influenzaimpfung allen Schwangeren ab dem 2. Trimenon der Schwangerschaft; bei einer erhöhten gesundheitlichen Gefährdung infolge eines Grundleidens schon ab dem 1. Trimenon [7]. Auch Menschen ab 60 Jahren sowie Personen mit chronischer Grunderkrankung und medizinisches Personal sollten die Impfung erhalten [8]. Die für Deutschland verfügbaren Studienergebnisse weisen jedoch auf eine geringe Inanspruchnahme der Impfung - insbesondere bei Schwangeren - hin $[9,10]$.
Um die Impfquoten zum Schutz vor der saisonalen Influenza zu erhöhen, wird seit 2006 regelmäßig die vom Bundesministerium für Gesundheit geförderte gemeinsame Kampagne der Bundeszentrale für gesundheitliche Aufklärung (BZgA) und des Robert Koch-Instituts (RKI) ,Wir kommen der Grippe zuvor" durchgeführt. Im Rahmen der Kampagne werden kostenlos Informationsmaterialien zur Verfügung gestellt. Auch gynäkologische Praxen erhalten in diesem Zusammenhang Medien, die sie zur Impfaufklärung nutzen können. Da die Schwangerenvorsorge primär durch niedergelassene Frauenärztinnen und -ärzte erfolgt [11], nehmen diese eine zentrale Rolle ein, um die Impfbereitschaft schwangerer Frauen zu erhöhen.

Auch die Impfung gegen humane Papillomaviren (HPV) wird in gynäkologischen Praxen durchgeführt. Durch diese Impfung soll die Krankheitslast durch Gebärmutterhalskrebs und seine Vorstufen - verursacht durch die Hochrisiko-HPV-Typen 16 und 18 verringert werden. Seit 2007 wird die Impfung für Mädchen im Alter von 12 bis 17 Jahren empfohlen [12]. 2014 wurde das für die Impfung empfohlene Alter auf 9 bis 14 Jahre vorgezogen, womit sich auch die erforderliche Anzahl an Impfdosen von 3 auf 2 reduziert [13]. Impfquoten für das 2-Dosen-Schema liegen aktuell noch nicht vor, fallen jedoch für eine Impfserie bestehend aus 3 Impfdosen eher gering aus. Sie lagen 2012 zwischen 1,2\% bei 12-jährigen Mädchen [14] und 36,2 bis $45,9 \%$ bei 16-jährigen Mädchen [14, 15].

Verschiedene Studien zeigen, dass die ärztliche Empfehlung ein wichtiger Prädiktor für eine positive Impfentscheidung ist [16-22]. Für Deutschland liegen bisher jedoch keine Daten aus bundesweit durchgeführten Erhebungen mit Gynäkologinnen und Gynäkologen vor, die die Analyse der Umsetzung der Impfempfehlungen für Influenza und HPV ermöglichen. Diese sind jedoch notwendig, um in zukünftigen Maßnahmen zur Steigerung der Impfquoten auch die Bedürfnisse dieser Facharztgruppe adäquat berücksichtigen und Barrieren zur Umsetzung der Impfempfehlungen aufdecken zu können.

Daher sollte folgenden Fragen nachgegangen werden: 1) Lassen sich Gynäkologinnen und Gynäkologen sowie ihr Praxispersonal regelmäßig gegen saisonale Influenza impfen? 2) Empfehlen Gynäkologinnen und Gynäkologen Schwangeren, aber auch chronisch Kranken und Senioren die Impfung gegen saisonale Influenza? Welche 


\begin{tabular}{|c|c|c|}
\hline & Studienkollektiv, \% & $\begin{array}{l}\text { Grundgesamtheit der } \\
\text { niedergelassenen Gynäkologinnen } \\
\text { und Gynäkologen, } \%(n=9798)^{a}\end{array}$ \\
\hline \multicolumn{3}{|l|}{ Geschlecht $(n=1458)$} \\
\hline Weiblich & 70,8 & 60,1 \\
\hline Männlich & 29,2 & 39,9 \\
\hline \multicolumn{3}{|l|}{ Altersgruppen $(n=1414)$} \\
\hline$\leq 39$ Jahre & 4,5 & 3,0 \\
\hline 40 bis 59 Jahre & 75,6 & 71,8 \\
\hline$\geq 60$ Jahre & 19,9 & 25,3 \\
\hline \multicolumn{3}{|l|}{ Bundesland $(n=1442)$} \\
\hline Ostdeutsche Bundesländer ${ }^{b}$ & 28,8 & 18,9 \\
\hline Westdeutsche Bundesländer & 71,2 & 81,1 \\
\hline
\end{tabular}

Gründe sprechen gegen eine Impfung? 3) Wie sieht das Informationsverhalten zur Influenzaimpfung aus? Darüber hinaus sollte ermittelt werden, ob 4) Gynäkologinnen und Gynäkologen jungen Mädchen und jungen Frauen die HPVImpfung empfehlen bzw. welche Gründe gegen eine Impfempfehlung sprechen.

\section{Methode}

Im Rahmen der nationalen InfluenzaImpfaufklärungskampagne „Wir kommen der Grippe zuvor" erfolgte vor Beginn der Influenzasaison im September 2014 eine Aussendung von Medienpaketen an niedergelassene Gynäkologinnen und Gynäkologen, Pädiater, Internisten, Allgemein- und Betriebsmediziner, an den öffentlichen Gesundheitsdienst, sozialpädiatrische Zentren, an Krankenhäuser sowie an Alten- und Pflegeheime. Die Grundgesamtheit der hier dargestellten Erhebung bildeten alle niedergelassenen Gynäkologinnen und Gynäkologen, deren Adressen über einen kommerziellen Anbieter zugänglich waren. Insgesamt wurden Medienpakete an 7477 niedergelassene Gynäkologinnen und Gynäkologen versandt. Dies waren $76,3 \%$ aller in Deutschland niedergelassenen Gynäkologinnen und Gynäkologen [23]. Neben Informationsmaterialien enthielt die Aussendung einen Fragebogen sowie ein Anschreiben, in dem über die Befragung aufgeklärt wurde. Der ausgefüllte Fragebogen konnte postalisch (über einen beigelegten frankierten Rückumschlag) oder per
Fax an das RKI anonym zurückgesandt werden. Es erfolgte keine Incentivierung der teilnehmenden gynäkologischen Praxen.

Der Fragebogen bestand überwiegend aus geschlossenen Fragen. Lediglich bei Empfehlung der Influenza- bzw. HPVImpfung sprechen, sollten die Befragten bei der Wahl der Antwortmöglichkeit „andere Gründe“ diese im Freitextfeld näher aufführen.

Die statistischen Analysen erfolgten mit dem Statistikprogramm StataSE13. Die Verteilungen der Ausprägungen kateHäufigkeiten mit $95 \%$-Konfidenzintervallen (KI) dargestellt, bivariate Assoziationen von kategorialen Variablen wurden mithilfe des $\chi^{2}$-Tests auf statistische Unabhängigkeit getestet. Ein $p$-Wert $<0,05$ wurde als statistisch signifikant angesehen. Fehlende Werte wurden als solche behandelt und gingen nicht in die Analysen ein. Die dargestellten Ergebnisse beziehen sich somit auf die Anzahl der Personen, die die jeweilige Frage beantworteten. Die Auswertung der Freitextfelder erfolgte auf Grundlage einer inhaltlichen Kategorisierung.

\section{Ergebnisse}

\section{Stichprobe}

Von den insgesamt 1484 eingegangenen Fragebögen konnten 1469 in die Datenanalysen einbezogen werden. Bei den den Fragen nach Gründen, die gegen eine gorialer Variablen wurden als prozentuale übrigen Fragebögen wurde ein unvollständiger Bogen - bestehend aus nur einer Seite - per Fax zurückgesandt. Dies spiegelt eine Response von 19,6\% wider. Der Großteil der ausgefüllten Bögen kam auf dem Postweg zurück $(91,2 \%)$. Nur wenige Ärztinnen und Ärzte (8,9\%) wählten das Fax. Die Charakteristika der teilnehmenden Gynäkologinnen und Gynäkologen sind in $\bullet$ Tab. 1 aufgeführt. Die Mehrheit der Teilnehmenden war weiblich. Im Median waren die Gynäkologinnen und Gynäkologen 52 Jahre alt (Spanne: 30-78 Jahre) und seit 14,5 Jahren (Spanne: 0,25-41 Jahre) als niedergelassene Ärztin bzw. als Arzt tätig.

\section{Impfung und Impfempfehlung gegen Influenza in der gynäkologischen Praxis}

Etwa drei Viertel der bundesweit befragten Ärztinnen und Ärzte $(72,2 \%, 95 \%$-KI: 69,8-74,5\%) gaben an, dass sie sich jedes Jahr gegen Influenza impfen lassen. Nur 16,7\% (95\%-KI: 14,8-18,7\%) ließen sich gelegentlich und 11,1\% (95\%-KI: 9,6$12,9 \%$ ) gar nicht gegen saisonale Influenza impfen. Während es in Bezug auf die Impfquote keine Unterschiede zwischen Alter, Geschlecht und Dauer der Niederlassung gab, waren die Gynäkologinnen und Gynäkologen in den ostdeutschen Bundesländern signifikant häufiger regelmäßig geimpft als in den westdeutschen Bundesländern ( 78,5 vs. $70,0 \%, p<0,05)$.

Ein Großteil der Befragten (94,2\%, 95\%-KI: 92,8-95,3\%) bot seinem Praxisteam die Influenzaimpfung an. Während etwa die Hälfte der teilnehmenden Ärztinnen und Ärzte angab, dass das Praxispersonal zum Teil geimpft sei (55,0\%, 95\%-KI: 52,4-57,6\%), bekräftigte ein Drittel der Teilnehmer (34,3\%, $95 \%$-KI: 31,8-36,8\%), dass das gesamte Praxispersonal gegen saisonale Influenza geimpft sei. Nur 8,1 \% (95\%-KI: 6,7-9,6\%) der Ärztinnen und Ärzte sagten, dass die Mitarbeiter nicht geimpft seien.

Es konnte gezeigt werden, dass dem Praxisteam signifikant häufiger eine Influenzaimpfung angeboten wurde, wenn die Befragten selbst regelmäßig oder gelegentlich die Influenzaimpfung in Anspruch genommen haben $(n=1199)$ als 
Bundesgesundheitsbl 2016 ·59:396-403 DOI 10.1007/s00103-015-2303-6

(c) Springer-Verlag Berlin Heidelberg 2015

\section{B. Bödeker · L. Seefeld · S. Buck · O. Ommen · O. Wichmann}

\section{Wie werden die Impfempfehlungen gegen saisonale Influenza und gegen humane Papillomaviren in gynäkologischen Praxen umgesetzt?}

\section{Zusammenfassung}

In Deutschland ist die Impfung gegen saisonale Influenza bei Schwangeren seit 2010 und die gegen humane Papillomaviren (HPV) bei Mädchen seit 2007 empfohlen. Niedergelassene Gynäkologinnen und Gynäkologen nehmen bei der Kommunikation und Impfung der beiden Zielgruppen eine wichtige Rolle ein. Darüber hinaus sollten auch medizinisches Personal sowie Menschen ab 60 Jahren und Personen mit chronischer Grunderkrankung die Impfung gegen saisonale Influenza erhalten. Ziel der vorliegenden Erhebung war es, einen ersten Einblick zu gewinnen, inwiefern die Influenza- und HPVImpfempfehlungen in gynäkologischen Praxen umgesetzt werden. Im Rahmen der vom Robert Koch-Institut (RKI) und der Bundeszentrale für gesundheitliche Auf- klärung (BZgA) gemeinsam durchgeführten Kampagne „Wir kommen der Grippe zuvor" wurde im September 2014 ein Fragebogen zusätzlich zu den Medienpaketen zur Influenzaimpfaufklärung bundesweit an 7477 niedergelassene Gynäkologinnen und Gynäkologen verschickt. Es wurden Daten aus 1469 (20\%) Praxen in die Analysen einbezogen. $72 \%$ der befragten Ärztinnen und Ärzte lassen sich jährlich gegen Influenza impfen. Die Mehrheit der Niedergelassenen empfahl Schwangeren die Impfung gegen saisonale Influenza (93\%) sowie Mädchen und jungen Frauen die HPV-Impfung (97\%). Von denjenigen, die keine Empfehlung aussprachen, wurden als Hauptgründe gegen die Influenzaimpfung Sicherheits- und gegen die HPV-Impfung Wirksamkeitsbedenken angegeben. Gegen beide Impfungen wurde zu- sätzlich aufgeführt, dass ein Aufklärungsgespräch zu aufwendig sei. Die in unserer Studienpopulation hohe Akzeptanz der beiden Impfungen spiegelt sich nicht in den für Deutschland verfügbaren Impfquoten wider. Gynäkologinnen und Gynäkologen müssen verstärkt auf ihre wichtige Rolle in der Impfprävention hingewiesen werden. Sowohl während der medizinischen Ausbildung als auch in weiterführenden gynäkologischen Fort- und Weiterbildungen sollte das Thema Impfen und die Kommunikation hierzu vermehrt berücksichtigt werden.

Schlüsselwörter

Influenzaimpfung · HPV-Impfung ·

Gynäkologie · Impfaufklärung ·

Informationsbedarf

\section{Implementation of seasonal influenza and human papillomavirus vaccination recommendations in gynecological practices in Germany}

\section{Abstract}

In Germany, seasonal influenza vaccination has been recommended for pregnant women since 2010 and human papillomavirus (HPV) vaccination for girls since 2007. Gynecologists play an important role in the communication and vaccination of these two target groups. Moreover, seasonal influenza vaccination is also recommended for healthcare workers, as well as adults aged $\geq 60$ years and individuals with underlying chronic diseases. The aim of this study was to gain first insights into the acceptance and implementation of the seasonal influenza und HPV vaccination recommendations in gynecological practices. In the context of the national influenza immunization campaign - which is jointly carried out by the Robert Koch Institute (RKI) and the Federal Centre for Health Education (BZgA) - a questionnaire was sent together with influenza information kits to 7477 gynecologists in September 2014. Data from 1469 (20\%) gynecologists were included in the analysis. $72 \%$ of respondents reported that they themselves received a seasonal influenza shot each year. The majority of gynecologists recommended seasonal influenza vaccination for pregnant women (93\%) and HPV vaccination for girls (97\%). The most commonly stated reasons against influenza vaccination were safety concerns. Those against HPV vaccination were effectiveness concerns. Additionally, for both vaccinations the provision of vaccine-related information to the patient was considered too time consum-
ing.The high acceptance of seasonal influenza and HPV vaccination among gynecologists is discordant with the available vaccination coverage figures in Germany. Gynecologists must be reminded of their important role in the prevention of vaccine-preventable diseases in adolescents and adult women. Immunization and communication skills should be considered more strongly as an integral part of medical education and further training for gynecologists.

Keywords

Influenza vaccination $\cdot$ HPV vaccination . Gynecology · Vaccine education · Information needs wenn diese nicht geimpft waren $(n=115)$ (91,3 vs. $8,8 \%, p<0,001)$.

Die klare Mehrheit der befragten Gynäkologinnen und Gynäkologen empfahl routinemäßig Frauen, die in der Influenzasaison schwanger sind, sich gegen Influenza impfen zu lassen (93,4\%, 95\%-KI: 92,0-94,6\%). Auch die generellen Influenza-Impfempfehlungen für Patientinnen mit einer chronischen Erkrankung, die nicht schwanger sind, sowie für Patientinnen ab 60 Jahren wurden von der Mehrzahl der Befragten ausgesprochen (chronisch Kranke: 90,7\%, $95 \%$-KI: 89,1-92,2\%; Personen $\geq 60$ Jahren: 91,0 \%, 95\%-KI: 89,4-92,4\%). In den ostdeutschen Bundesländern wurden diese Impfempfehlungen signifikant häufiger geäußert als in den westdeutschen Bundesländern (Impfempfehlung für Schwangere: 95,9 vs. $92,7 \%, p<0,05$; Impfempfehlung für chronisch Kranke:
96,1 vs. $89,0 \%, p<0,001$; Impfempfehlung für Personen $\geq 60$ Jahren: 96,6 vs. $89,0 \%$, $p<0,001)$.

Die Influenza-Impfempfehlung für Schwangere, chronisch Kranke oder Personen $\geq 60$ Jahren wurde signifikant häufiger von Gynäkologinnen und Gynäkologen ausgesprochen, die selbst regelmäßig oder gelegentlich geimpft waren, als von teilnehmenden Ärztinnen und Ärzten, die nicht geimpft waren 


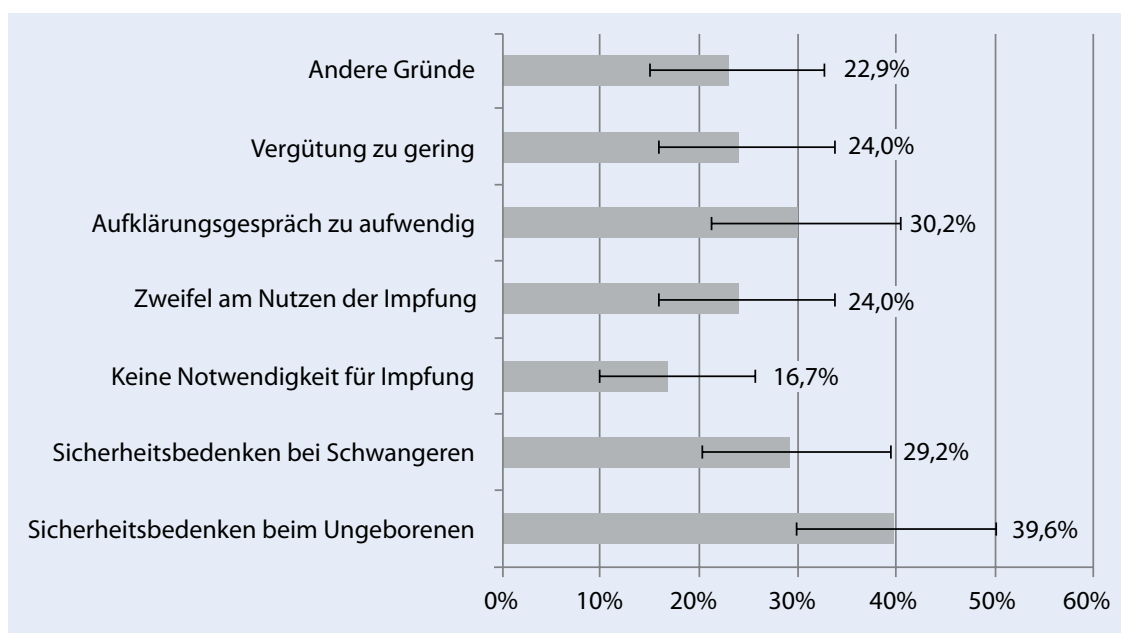

Abb. $1 \Delta$ Gründe, die nach Angaben der Gynäkologinnen und Gynäkologen gegen die InfluenzaImpfempfehlung bei Schwangeren sprachen $(n=96)$

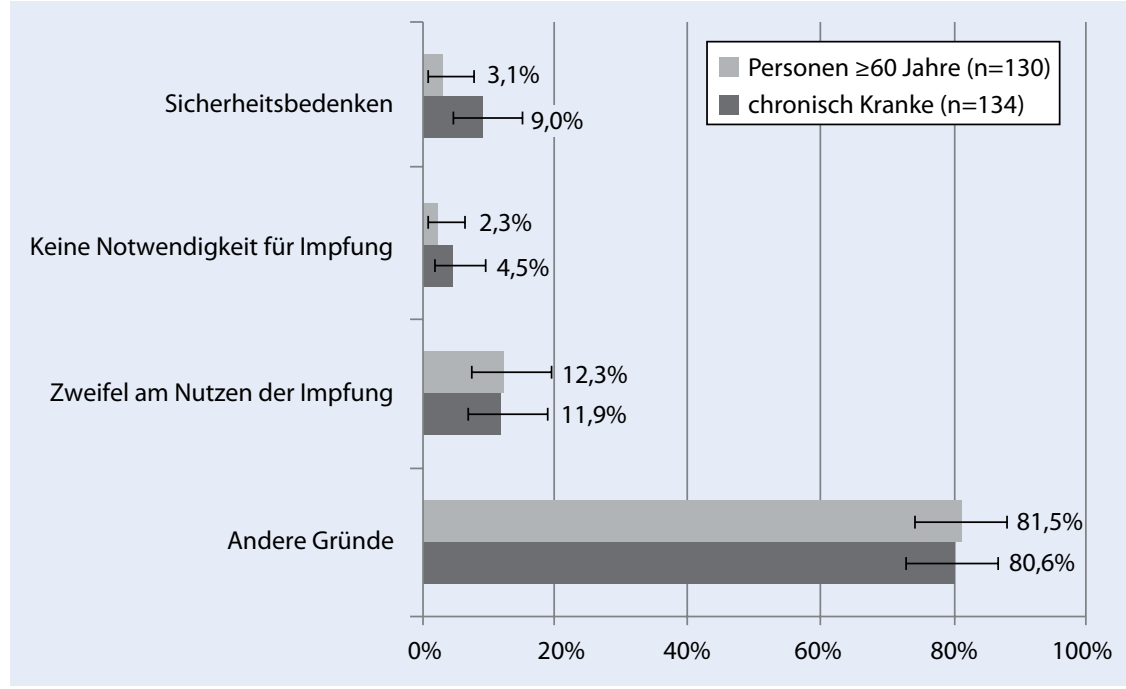

Abb. $2 \Delta$ Gründe, die nach Angaben der Gynäkologinnen und Gynäkologen gegen die InfluenzaImpfempfehlung bei Personen $\geq 60$ Jahren und chronisch Kranken sprachen

(Impfempfehlung für Schwangere: 91,5 vs. $8,5 \%$; Impfempfehlung für chronisch Kranke: 91,5 vs. $8,5 \%$; Impfempfehlung für Personen $\geq 60$ Jahren: 91,4 vs. $8,6 \%$, jeweils $p<0,001)$.

Die Gründe, die aus Sicht der Ärztin bzw. des Arztes gegen die jeweilige Influenza-Impfempfehlungen sprachen, sind in $\bullet$ Abb. 1 und 2 aufgeführt. Die von den Gynäkologinnen und Gynäkologen am häufigsten genannten Gründe, warum sie schwangeren Frauen keine Impfung empfahlen, waren „Bedenken wegen der Sicherheit des Impfstoffs beim ungeborenen Kind und bei der Schwangeren" sowie die Auffassung, dass der „Aufwand eines notwendigen Auf-

\section{Informationsverhalten zur Influenzaimpfung}

90,8 \% (95\%-KI: 89,1-92,3\%) der teilnehmenden Gynäkologinnen und Gynäkologen gaben an, dass sie in ihrer Praxis Informationsmaterialien zur Influenzaimpfung für die Beratung ihrer Patientinnen nutzen. Insgesamt äußerten mehr als ein Viertel der Befragten (29,0\%, $95 \%$-KI: $26,6-31,5 \%)$ weiteren eigenen Informationsbedarf zur Influenzaimpfung. Die gewünschten Themenbereiche sind in $\bullet$ Abb. 3 dargestellt. Der größte Informationsbedarf bestand demnach zu „möglichen Nebenwirkungen der Impfung“ und „Risiken bzw. Komplikationen einer Influenzaerkrankung". Etwa drei Viertel der Befragten $(76,1 \%, 95 \%$-KI: 71,6-80,2\%), die Informationsbedarf äußerten, gaben den Postweg als geeigneten Informationsweg an, gefolgt von E-Mail-Newslettern von z. B. Fachverbänden (18,9\%, $95 \%$-KI: 15,2-23,1\%), den Internetseiten des RKI (18,6\%, $95 \%$-KI: $14,9-22,8 \%)$, Fachtagungen (14,1\%, 95\%-KI: 10,8-17,9\%) und der Fachpresse (13,4\%, $95 \%$-KI: 10,2$17,1 \%)$.

\section{Impfempfehlung und Impfangebot gegen HPV}

Fast alle Gynäkologinnen und Gynäkologen (97,4\%, $95 \%$-KI: 96,4-98,1\%) gaben an, dass sie regelmäßig Mädchen und jungen Frauen die Impfung gegen HPV anbieten bzw. die Mütter auf die empfohlene Impfung ihrer Tochter hinweisen. Nur 2,0 \% (95\%-KI: 1,3-2,9\%) erwiderten, dass sie dies nur auf Nachfrage der Mutter bzw. des Mädchens tun. Lediglich 0,6\% (95\%-KI: 0,3-1,2\%) gaben an, dass sie die Impfung üblicherweise nicht anbieten. Die Gründe, warum die HPVImpfung nur auf Nachfrage oder gar nicht angeboten wurde ( $n=38$ Gynäkologinnen und Gynäkologen), sind in • Abb. 4 aufgeführt. Die am häufigsten genannten Gründe waren die Auffassung, dass das "Aufklärungsgespräch zu aufwendig" sei, „Zweifel am Nutzen der Impfung“ bestünden sowie die „Vergütung für das Aufklärungsgespräch und die Impfung zu gering" sei. 


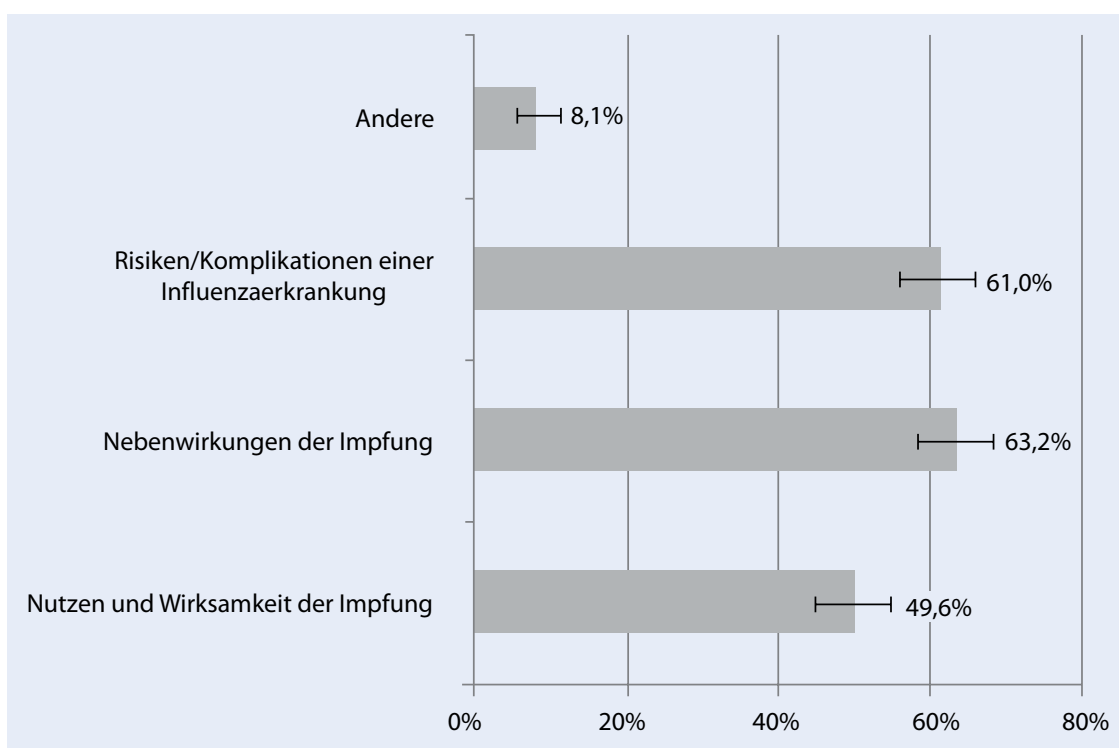

Abb. $3 \Delta$ Von Gynäkologinnen und Gynäkologen genannter Informationsbedarf nach Themenbereichen zur Influenzaimpfung $(n=397)$

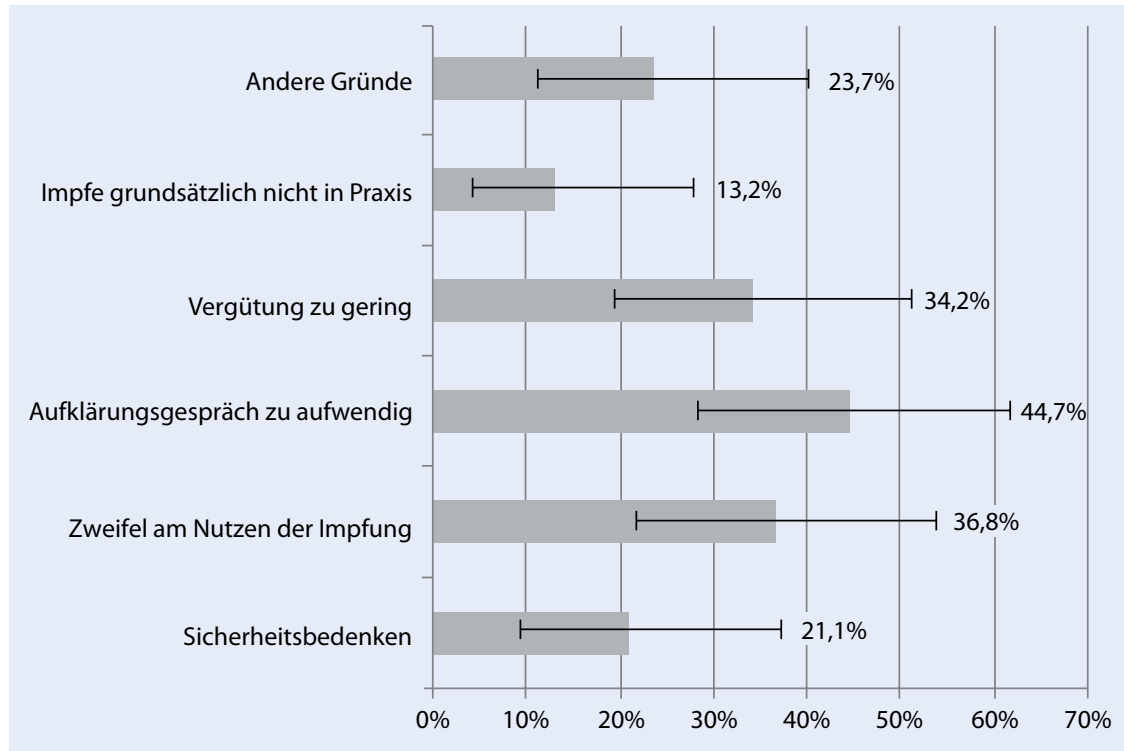

Abb. 4 \ Von Gynäkologinnen und Gynäkologen genannte Gründe, warum die HPV-Impfung nicht jungen Mädchen und jungen Frauen angeboten wurde bzw. dies nur auf Nachfrage erfolgte $(n=38)$

Es konnte weiterhin gezeigt werden, dass die HPV-Impfung häufiger von Gynäkologinnen und Gynäkologen angeboten wurde, die auch schwangeren Frauen eine Influenzaimpfung empfahlen ( $n=1321)$, als von Ärztinnen und Ärzten, die Schwangere nicht auf die Influenzaimpfung hinwiesen $(n=80)$ (94,3 vs. $5,7 \%, p<0,001)$. fragten Ärztinnen und Ärzte befürwortet sowohl die saisonale Influenzaimpfung Schwangerer als auch die HPV-Impfung von Mädchen und jungen Frauen.

Andere in Deutschland durchgeführte Studien belegen bei medizinischem Personal sehr geringe Influenzaimpfquoten. Diese lagen - unabhängig vom stationären bzw. ambulanten Sektor und ohne weitere Differenzierung des medizinischen Personals - je nach Saison zwischen 26 und $31 \%$ [24]. In weiteren Studien, in denen ausschließlich niedergelassene Ärztinnen und Ärzte befragt wurden, konnten jedoch höhere Impfquoten zwischen 61 und $73 \%$ nachgewiesen werden [25-27]. Diese Daten sind vergleichbar mit den hier vorliegenden Ergebnissen, gemäß derer sich $72 \%$ der Gynäkologinnen und Gynäkologen regelmäßig gegen saisonale Influenza impfen lassen. Es ist zu vermuten, dass niedergelassene Ärztinnen und Ärzte im Vergleich zu in Krankenhäusern tätigen Kolleginnen und Kollegen und anderem medizinischen Personal häufiger geimpft sind, um mögliche Arbeitsausfälle und den damit einhergehenden Verdienstausfall in der Praxis zu vermeiden [25]. Des Weiteren behandeln niedergelassene Ärztinnen und Ärzte in der Regel auch mehr Patientinnen und Patienten, die an Atemwegserkrankungen leiden, als die ärztlichen Kolleginnen und Kollegen im stationären Bereich, sodass hier vermutlich das angenommene eigene Infektionsrisiko ebenfalls zu höheren Impfquoten führt.

Darüber hinaus konnte gezeigt werden, dass die Influenza-Impfempfehlung der STIKO für Schwangere von den teilnehmenden Gynäkologinnen und Gynäkologen in einem hohen Maß akzeptiert wird: $93 \%$ empfahlen ihren schwangeren Patientinnen die Impfung. Diese positive Einstellung wird auch in Studien aus den USA, Kanada und Australien belegt. Demnach empfehlen in diesen Ländern 63 bis $89 \%$ der Gynäkologinnen und Gynäkologen den von ihnen betreuten Schwangeren die Impfung [2831]. Obwohl Studien zeigen, dass die ärztliche Empfehlung für die Influenzaimpfung ein sehr wichtiger Prädiktor für eine positive Impfentscheidung 
Schwangerer ist $[9,16]$, spiegelt sich die in unserer Erhebung nachgewiesene positive Einstellung der Befragten nicht in der Influenzaimpfquote für ihre Patientengruppe wider. Diese lag bei in Deutschland lebenden Schwangeren in der Influenzasaison 2012/13 bei lediglich $23 \%$ [9]. Die Diskrepanz zwischen den von Gynäkologinnen und Gynäkologen ausgesprochenen Impfempfehlungen und der tatsächlichen Impfquote Schwangerer zeigt sich auch in anderen internationalen Studien [29]. Eine mögliche Erklärung für diese Diskrepanz könnte in der Formulierung unserer Frage liegen, da nicht nach der tatsächlichen Durchführung der Impfung gefragt wurde. Es kann somit nicht ausgeschlossen werden, dass die Befragten die Impfung zwar generell befürworten, sie die Empfehlung aber nicht bei jeder Schwangeren konkret ansprechen und sie entsprechend durchführen. So konnten auch Panda et al. [30] zeigen, dass ein Großteil der Gynäkologinnen und Gynäkologen die Impfung empfiehlt, aber nur die Hälfte die Impfung im Praxisalltag auch wirklich anbietet. Ein weiterer Grund für die Diskrepanz zwischen der hohen Akzeptanz der Impfung unter der teilnehmenden Ärzteschaft und den tatsächlich erreichten Impfquoten muss in dem möglichen Selektionsbias unseres Studiensamples vermutet werden, auf den in den Studienlimitationen näher eingegangen wird.

Ähnlich zur Influenza-Impfempfehlung bei Schwangeren spiegelt sich auch bei der HPV-Impfung von Mädchen und jungen Frauen bei den teilnehmenden Gynäkologinnen und Gynäkologen eine sehr hohe Impfakzeptanz wider. Diese positive Einstellung zeigte sich auch in einer anderen in Deutschland und weiteren in den USA durchgeführten Studien, nach denen die große Mehrheit der Gynäkologinnen und Gynäkologen die HPV-Impfung empfahl und anbot. Obwohl auch bei der HPVImpfung die ärztliche Empfehlung ein wichtiger Faktor für eine positive Impfentscheidung ist $[17,31]$ und ein enger Zusammenhang zwischen der HPVImpfung und einem bereits erfolgten Frauenarztbesuch besteht $[14,15]$, fallen die HPV-Impfquoten der Mädchen trotz positiver Einstellung der Gynäkologinnen und Gynäkologen in Deutschland gering aus. Über einen kompletten Impfschutz verfügen demnach zwischen 36 und $46 \%$ der 16-jährigen Mädchen [14, 15]. Analog zur Influenzaimpfung Schwangerer muss als ein möglicher Grund für diese Diskrepanz ein Selektionsbias der Befragten angenommen werden. Die Differenz zwischen der positiven Einstellung gefragter Ärztinnen und Ärzte und den erreichten Impfquoten zeigte sich auch in Befragungen in den USA [32]. Weitere internationale Studien schlussfolgern, dass insbesondere bei der HPV-Impfung zusätzliche Faktoren berücksichtigt werden müssen, die den Zusammenhang zwischen der Impfempfehlung durch den Arzt und der finalen Impfentscheidung beeinflussen: Insbesondere die von den Eltern wahrgenommenen Sicherheitsbedenken der Impfung und die Annahme, die Impfung sei nicht notwendig, spielen eine wichtige Rolle [33, 34].

Neben gynäkologischen führen auch pädiatrische Praxen die Impfung von Mädchen gegen HPV durch. Durch die 2014 erfolgte Vorverlegung des Impfalters auf 9-14 Jahre kommt den pädiatrischen Praxen eine wichtigere Rolle bei der Prävention des Gebärmutterhalskrebses und deren Vorstufen zu. Die in Deutschland durchgeführten kindlichen bzw. jugendlichen Vorsorgeuntersuchungen U11 (im Alter von 9 bis 10 Jahren) bzw. J1 (im Alter von 12 bis 14 Jahren), die überwiegend von Kinder- und Jugendärztinnen und -ärzten durchgeführt werden und deren Teilnahmequoten insbesondere zur J1 immer noch zu gering ausfallen $[14,35]$, sollten verstärkt für die Durchführung der HPVImpfung genutzt werden. Trotzdem bedarf es auch einer Steigerung der Impfaktivitäten durch gynäkologische Praxen, insbesondere um den nicht durch die Vorsorgeuntersuchung erreichten jugendlichen Mädchen einen Impfschutz gegen HPV und den damit assoziierten Krankheiten zukommen zu lassen.

Eine Limitation der durchgeführten Studie ist die Response von lediglich $20 \%$. Somit beziehen sich die vorliegenden Daten auf lediglich etwa $15 \%$ aller in Deutschland niedergelassenen Gynäkologinnen und Gynäkologen. Aus anderen in Deutschland durchgeführten Studien mit niedergelassenen Ärztinnen und
Ärzten ist jedoch bekannt, dass diese eine schwer erreichbare Zielgruppe darstellen, sodass die Rücklaufquoten zwischen 9 und maximal $55 \%$ schwanken $[25,26$, 36]. Im Vergleich zu anderen empirischen Studien ist die Ausschöpfungsquote bei schriftlichen Befragungen ohne Bereitstellung eines Incentives, wie es auch hier umgesetzt wurde, grundsätzlich eher gering, sodass eine Response von über $20 \%$ nur selten erreicht wird [37]. Wie Studien aus Kanada und den USA zeigen [28, 38-40], kann die verhältnismäßig geringe Rücklaufquote auch ein Hinweis dafür sein, dass viele Gynäkologinnen und Gynäkologen Impfungen immer noch eher außerhalb ihres Aufgaben- und Verantwortungsbereiches sehen.

Aufgrund der insgesamt geringen Response kann ein Selektionsbias nicht ausgeschlossen werden. Es ist möglich, dass insbesondere jene Gynäkologinnen und Gynäkologen an der Erhebung teilnahmen, die sowohl der Influenzaals auch der HPV-Impfung gegenüber positiv eingestellt waren, wodurch die Influenzaimpfquoten und die ärztliche Impfakzeptanz überschätzt wurden. Darüber hinaus macht auch der Vergleich der Studienteilnehmer mit der Grundgesamtheit der bundesweit tätigen niedergelassenen Gynäkologinnen und Gynäkologen deutlich (• Tab. 1), dass gemessen an der Grundgesamtheit der Niedergelassenen insgesamt mehr Ärztinnen und Ärzte aus den ostdeutschen als aus den westdeutschen Bundesländern teilnahmen. In verschiedenen Studien - wie auch in unserer - konnte gezeigt werden, dass in den ostdeutschen Bundesländern verglichen mit den westdeutschen eine positivere Grundeinstellung zum Thema Impfen herrscht, und somit sowohl in der Allgemeinbevölkerung [10, 18] als auch bei Ärztinnen und Ärzten [25, 26] höhere Impfquoten erreicht werden. Das selektierte Studiensample und mögliche Verzerrungen durch ein sozial erwünschtes Antwortverhalten der Frauenärztinnen und -ärzte können Gründe für die insgesamt sehr positiven Einstellungen zu den Influenza- und HPVImpfempfehlungen der STIKO darstellen. Obwohl die Effekte sozialer Erwünschtheit bei schriftlich-postalischen Befragungen im Vergleich zu persönlichen 
oder telefonischen Interviews geringer ausgeprägt sind, lässt sich ein sozial erwünschtes Antwortverhalten nicht gänzlich ausschließen [41, 42]. Insbesondere müssen einige Ergebnisse, wie z. B. zu den Gründen, warum die Impfungen nicht empfohlen werden, aufgrund der geringen Zahl an nicht-impfenden Ärztinnen und Ärzten im Studiensample, mit Vorsicht interpretiert werden.

Insgesamt lässt sich festhalten, dass trotz der dargestellten Limitationen davon ausgegangen werden kann, dass eine generelle Bereitschaft und Akzeptanz der Ärzteschaft besteht, die Influenza- und HPV-Impfung Schwangeren bzw. jungen Mädchen zu empfehlen. Dies stellt eine wichtige Grundvoraussetzung dar, um die Impfquoten in der entsprechenden Zielpopulation zu erhöhen. In diesem Kontext sollten zielgruppenspezifische Maßnahmen sowohl für Gynäkologinnen und Gynäkologen als auch für die Bevölkerungsgruppen, denen eine Impfung empfohlen wird, durchgeführt werden. Es ist zum Beispiel denkbar, dass aufgrund des existierenden Zeitmangels der Ärztinnen und Ärzte, Sprechstundenhilfen bereits zu Beginn des Arztbesuches Schwangere und junge Mädchen oder ihre Mütter auf die entsprechende Impfung hinweisen und beratend zur Seite stehen. Aber auch durch die Anwendung von Erinnerungssystemen können Impfquoten gesteigert werden $[26,43]$.

Die Befürwortung der Impfempfehlungen muss vonseiten der Gynäkologinnen und Gynäkologen in der Praxis noch aktiver umgesetzt werden. In diesem Kontext muss diese Facharztgruppe verstärkt auf ihre wichtige Rolle in der Impfprävention hingewiesen werden. Es sollte bereits während der medizinischen Ausbildung, aber auch kontinuierlich nach Beendigung des Medizinstudiums und der Facharztausbildung das Thema Impfen vermehrt in gynäkologischen Fort- und Weiterbildungen berücksichtigt werden. Durch die regelmäßigen medizinischen Untersuchungen im Rahmen der Schwangerenvorsorge haben Gynäkologinnen und Gynäkologen den direkten und kontinuierlichen Kontakt zu dieser Zielgruppe und können in diesem Rahmen die saisonale Influenzaimpfung aktiv an- bieten. Unabhängig von der Influenzaimpfung sollten Gynäkologinnen und Gynäkologen bei Frauen im gebärfähigen Alter und bei Frauen mit Kinderwunsch auf einen vollständigen Impfschutz insbesondere gegen Masern, Röteln, Pertussis und Varizellen achten. Aber auch für die Schließung von Impflücken bei jugendlichen Mädchen und jungen Frauen in Bezug auf die HPV-Impfung kommt den Gynäkologinnen und Gynäkologen eine äußerst wichtige Rolle zu. Erste Kontakte von jugendlichen Mädchen sollten dazu genutzt werden, den Impfstatus bzgl. der HPV-Impfung zu kontrollieren und fehlende Impfungen zu verabreichen.

\section{Korrespondenzadresse}

\section{B. Bödeker}

Fachgebiet Impfprävention, Abteilung für Infektionsepidemiologie,

Robert Koch-Institut

Seestraße 10,13353 Berlin

BoedekerB@rki.de

Danksagung. Wir danken den Gynäkologinnen und Gynäkologen, die an der Befragung teilgenommen haben, sowie dem Berufsverband der Frauenärzte, der uns in der Vorbereitungsphase der Studie für fachliche Fragen zur Verfügung stand.

\section{Einhaltung ethischer Richtlinien}

Interessenkonflikt. B. Bödeker, L. Seefeld, S. Buck, 0 . Ommen und $\mathrm{O}$. Wichmann geben an, dass kein Interessenkonflikt besteht.

Dieser Beitrag enthält keine Studien an Menschen oder Tieren.

\section{Literatur}

1. Rasmussen SA, Jamieson DJ, Uyeki TM (2012) Effects of influenza on pregnant women and infants. Am J Obstet Gynecol 207:S3-S8

2. Ortiz JR, Englund JA, Neuzil KM (2011) Influenza vaccine for pregnant women in resourceconstrained countries: a review of the evidence to inform policy decisions. Vaccine 29:4439-4452

3. Omer SB, Goodman D, Steinhoff MC et al (2011) Maternal influenza immunization and reduced likelihood of prematurity and small for gestational age births: a retrospective cohort study. PLoS Med 8:e1000441

4. Sheffield JS, Greer LG, Rogers VL et al (2012) Effect of influenza vaccination in the first trimester of pregnancy. Obstet Gynecol 120:532-537

5. Zaman K, Roy E, Arifeen SE et al (2008) Effectiveness of maternal influenza immunization in mothers and infants. N Engl J Med 359:15551564
6. Keller-Stanislawski B, Englund JA, Kang G et al (2014) Safety of immunization during pregnancy: a review of the evidence of selected inactivated and live attenuated vaccines. Vaccine 32:70577064

7. Robert Koch-Institut (2010) Mitteilung der Ständigen Impfkommission (STIKO) am Robert Koch-Institut: Änderung der Empfehlung zur Impfung gegen Influenza. Epid Bulletin 31:299309

8. Robert Koch-Institut (2014) Empfehlungen der Ständigen Impfkommission (STIKO) am Robert Koch-Institut/Stand: August 2014. Epid Bulletin 34:305-340

9. Bödeker B, Walter D, Reiter S, Wichmann O (2014) Cross-sectional study on factors associated with influenza vaccine uptake and pertussis vaccination status among pregnant women in Germany. Vaccine 32:4131-4139

10. Bödeker B, Remschmidt C, Müters S, Wichmann O (2015) Impfquoten unter Erwachsenen in Deutschland für die Impfungen gegen saisonale Influenza, Tetanus und Pertussis. Bundesgesundheitsblatt Gesundheitsforschung Gesundheitsschutz 58:174-181

11. Vetter K, Goeckenjan M (2013) Schwangerenvorsorge in Deutschland. Bundesgesundheitsblatt Gesundheitsforschung Gesundheitsschutz 56:1679-1685

12. Robert Koch-Institut (2007) Mitteilung der Ständigen Impfkommission (STIKO) am Robert Koch-Institut: Impfung gegen humane Papillomaviren (HPV) für Mädchen von 12 bis 17 Jahren Empfehlung und Begründung 2007. Epid Bulletin 12:97-103

13. Robert Koch-Institut (2014) Mitteilung der Ständigen Impfkommission am Robert KochInstitut: Empfehlungen der Ständigen Impfkommission (STIKO) am Robert Koch-Institut/ Stand: August 2014. Epid Bulletin 34:305-340

14. Rieck T, Feig M, Delere Y, Wichmann O (2014) Utilization of administrative data to assess the association of an adolescent health check-up with human papillomavirus vaccine uptake in Germany. Vaccine 32:5564-5569

15. Poethko-Müller C, Buttmann-Schweiger N (2014) Impfstatus und Determinanten der Impfung gegen humane Papillomviren (HPV) bei Mädchen in Deutschland. Bundesgesundheitsblatt Gesundheitsforschung Gesundheitsschutz 57:869-877

16. Yuen CY, Tarrant M (2014) Determinants of uptake of influenza vaccination among pregnant women - a systematic review. Vaccine 36:4602-4613

17. Kessels SJ, Marshall HS, Watson M, Braunack-Mayer AJ, Reuzel R, Tooher RL (2012) Factors associated with HPV vaccine uptake in teenage girls: a systematic review. Vaccine 30:3546-3556

18. Böhmer MM, Walter $D$, Krause $G$, Muters $S$, Gosswald A, Wichmann O (2011) Determinants of tetanus and seasonal influenza vaccine uptake in adults living in Germany. Hum Vaccin 7:1317-1325

19. Streng A, Seeger K, Grote V, Liese JG (2010) Varicella vaccination coverage in Bavaria (Germany) after general vaccine recommendation in 2004. Vaccine 28:5738-5745

20. Williams WW, Lu PJ, O'Halloran A et al (2015) Vaccination coverage among adults, excluding influenza vaccination - United States, 2013. MMWR Morb Mortal Wkly Rep 64:95-102 
21. Schneeberg A, Bettinger JA, McNeil S et al (2014) Knowledge, attitudes, beliefs and behaviours of older adults about pneumococcal immunization, a Public Health Agency of Canada/Canadian Institutes of Health Research Influenza Research Network (PCIRN) investigation. BMC Public Health $14: 442$

22. Bundeszentrale Für Gesundheitliche Aufklärung (2013) Einstellungen, Wissen und Verhalten der Allgemeinbevölkerung zu Hygiene und Infektionsschutz. http://www.bzga.de/forschung/studienuntersuchungen/studien/impfen-und-hygiene/ ?sub=79. Zugegriffen: 7. Apr. 2015

23. Gesundheitsberichterstattung Des Bundes (2013) Niedergelassene Ärztinnen und Ärzte mit Gebiets- und Facharztbezeichnung Frauenheilkunde und Geburtshilfe in 2013. https:// www.gbe-bund.de/gbe10/hrecherche.prc_herkunft_rech?tk=51310\&tk2 $=51312 \& p \_f i d=462 \& p$ uid $=$ gast $\&$ p_aid $=63901526 \& p \_s p r a c h e=D \& c n t \_$ $\mathrm{ut}=1 \& \mathrm{ut}=51312$. Zugegriffen: 26 . März 2015

24. Böhmer MM, Walter D, Falkenhorst $G$, Muters S, Krause G, Wichmann O (2012) Barriers to pandemic influenza vaccination and uptake of seasonal influenza vaccine in the post-pandemic season in Germany. BMC Public Health 12:938

25. Böhmer MM, Walter D, Ehrhardt J, Reiter S, Krause G, Wichmann O (2014) Saisonale und pandemische Influenzaimpfung: Impfquoten und Einstellung niedergelassener Ärzte in Deutschland. Gesundheitswesen 76:44-47

26. Wortberg S, Walter D (2010) Erinnerungssysteme zur Erhöhung der Impfrate gegen saisonale Influenza bei Risikopatienten. Eine Befragung niedergelassener Ärzte. Dtsch Med Wochenschr 135:1113-1117

27. Wortberg S, Walter D, Von Dem Knesebeck M, Reiter S (2009) Niedergelassene Ärzte als Multiplikatoren der Influenzaimpfung bei älteren Menschen, chronisch Kranken und medizinischem Personal. Bundesgesundheitsblatt Gesundheitsforschung Gesundheitsschutz 52:945-952

28. Tong $A$, Biringer $A$, Ofner-Agostini $M$, Upshur R, McGeer A (2008) A cross-sectional study of maternity care providers' and women's knowledge, attitudes, and behaviours towards influenza vaccination during pregnancy. J Obstet Gynaecol Can 30:404-410

29. Lu AB, Halim AA, Dendle C et al (2012) Influenza vaccination uptake amongst pregnant women and maternal care providers is suboptimal. Vaccine 30:4055-4059

30. Panda B, Stiller R, Panda A (2011) Influenza vaccination during pregnancy and factors for lacking compliance with current CDC guidelines. J Matern Fetal Neonatal Med 24:402-406

31. Remschmidt C, Walter D, Schmich P, Wetzstein M, Delere Y, Wichmann O (2014) Knowledge, attitude, and uptake related to human papillomavirus vaccination among young women in Germany recruited via a social media site. Hum Vaccin Immunother 10:2527-2535

32. Young JL, Bernheim RG, Korte JE, Stoler MH, Guterbock TM, Rice LW (2011) Human papillomavirus vaccination recommendation may be linked to reimbursement: a survey of Virginia family practitioners and gynecologists. J Pediatr Adolesc Gynecol 24:380-385

33. Feemster KA, Middleton $M$, Fiks AG, Winters $S$, Kinsman SB, Kahn JA (2014) Does intention to recommend $H P V$ vaccines impact $H P V$ vaccination rates? Hum Vaccin Immunother 10:2519-2526
34. Darden PM, Thompson DM, Roberts JR et al (2013) Reasons for not vaccinating adolescents: national immunization survey of teens, 2008-2010. Pediatrics 131:645-651

35. Hagen B, Strauch S (2011) The J1 adolescent health check-up: analysis of data from the German KiGGS survey. Dtsch Arztebl Int 108:180-186

36. Takla A, Wichmann O, Koch J, Terhardt M, Hellenbrand W (2014) Survey of pediatricians in Germany reveals important challenges for possible implementation of meningococcal $B$ vaccination. Vaccine 32:6349-6355

37. Diekmann A (1995) Empirische Sozialforschung: Grundlagen, Methoden, Anwendungen. Rowohlt, Hamburg, S 441

38. Kissin DM, Power ML, Kahn EB et al (2011) Attitudes and practices of obstetriciangynecologists regarding influenza vaccination in pregnancy. Obstet Gynecol 118:1074-1080

39. Link-Gelles R, Chamberlain AT, Schulkin J et al (2012) Missed opportunities: a national survey of obstetricians about attitudes on maternal and infant immunization. Matern Child Health J 16:1743-1747

40. Jessop AB, Dumas H, Moser CA (2013) Delivering influenza vaccine to high-risk adults: subspecialty physician practices. Am J Med Qual 28:232-237

41. Lamnek S, Schäfer WJ (1998) Befragungsmethoden reviewed: face-to-face, telefonisch oder postalisch? Sozialwissenschaften Berufspraxis 21:162-171

42. Gilkey MB, Moss JL, Coyne-Beasley T, Hall ME, Shah PD, Brewer NT (2015) Physician communication about adolescent vaccination: how is human papillomavirus vaccine different? Prev Med 77:181-185

43. Shavell VI, Moniz MH, Gonik B, Beigi RH (2012) Influenza immunization in pregnancy: overcoming patient and health care provider barriers. Am J Obstet Gynecol 207:67-74 
